\title{
Author Correction: Down-regulated GATA-1 up-regulates interferon regulatory factor 3 in lung adenocarcinoma
}

\author{
Lu-Lu Wang ${ }^{1}$, Zheng-Sen Chen ${ }^{2}$, Wen-Di Zhou ${ }^{3}$, Jin Shu ${ }^{4}$, Xiao-Hua Wang ${ }^{5}$, Rui Jin ${ }^{1}$, \\ Li-Li Zhuang ${ }^{1}$, Mir Alireza Hoda ${ }^{6}$, Hao Zhang $\mathbb{D}^{7}$ \& Guo-Ping Zhou ${ }^{1}$
}

Correction to: Scientific Reports https://doi.org/10.1038/s41598-017-02700-5, published online 31 May 2017

The original version of this Article contained an error in the order of corresponding authors. This error has now been corrected in the PDF and HTML versions of the Article.

(i) Open Access This article is licensed under a Creative Commons Attribution 4.0 International License, which permits use, sharing, adaptation, distribution and reproduction in any medium or format, as long as you give appropriate credit to the original author(s) and the source, provide a link to the Creative Commons license, and indicate if changes were made. The images or other third party material in this article are included in the article's Creative Commons license, unless indicated otherwise in a credit line to the material. If material is not included in the article's Creative Commons license and your intended use is not permitted by statutory regulation or exceeds the permitted use, you will need to obtain permission directly from the copyright holder. To view a copy of this license, visit http://creativecommons.org/licenses/by/4.0/.

(C) The Author(s) 2018

\footnotetext{
${ }^{1}$ Dpartment of Pediatrics, The First Affiliated Hospital, Nanjing Medical University, Nanjing, Jiangsu Province, China. ${ }^{2}$ Department of Urology, The Second Affiliated Hospital, Nanjing Medical University, Nanjing, Jiangsu Province, China. ${ }^{3}$ Dpartment of Pediatrics, Huai'an First People's Hospital, Nanjing Medical University, Huai'an, Jiangsu Province, China. ${ }^{4}$ Department of Pediatric Respiration, Affiliated Wuxi People's Hospital, Nanjing Medical University, Wuxi, Jiangsu Province, China. ${ }^{5}$ Dpartment of Pediatrics, Nanjing First Hospital, Nanjing Medical University, Nanjing, Jiangsu Province, China. ${ }^{6}$ Translational Thoracic Oncology Laboratory, Division of Thoracic Surgery, Department of Surgery, Comprehensive Cancer Center, Medical University Vienna, Vienna, Austria. ${ }^{7}$ Department of Thoracic and Cardiovascular Surgery, Affiliated Hospital of Xuzhou Medical University, Xuzhou, Jiangsu Province, China. Correspondence and requests for materials should be addressed to G.-P.Z. (email: guopingzhou@aliyun.com) or H.Z. (email: zhanghao@xzhmu.edu.cn)
} 